\title{
Síndrome metabólico y antipsicóticos de segunda generación.
}

Metabolic syndrome and second generation antipsychotic agents.

\author{
Benjamín Cortés Morales ${ }^{\mathrm{a}}$.
}

${ }^{a}$ Psiquiatra. Servicio de Psiquiatría, Hospital Universitario de Salamanca.

Correspondencia: Benjamín Cortés Morales (bmorales@saludcastillayleon.es)

Recibido: 12/09/2010; aceptado con modificaciones: 16/01/2011

RESUMEN: Aunque algunos antipsicóticos de segunda generación reducen el riesgo de efectos adversos extrapiramidales frente a los de primera generación, su uso se ha asociado a la aparición de síndrome metabólico. Esta es una entidad caracterizada por resistencia a la insulina, obesidad abdominal, dislipidemia aterogénica (hipertrigliceridemia, disminución del colesterolHDL y aumento del colesterol-LDL), hipertensión arterial y un estado protrombótico y proinflamatorio. El síndrome metabólico puede aumentar significativamente el riesgo de enfermedad cardiovascular y muerte prematura por lo que su abordaje multidisciplinar podría incidir sustancialmente en el estado de salud general de los pacientes que lo desarrollen.

Se presenta en este trabajo una revisión de la asociación del síndrome metabólico y los antipsicóticos de segunda generación. Se revisan hipótesis y hallazgos actuales en la fisiopatología, el aumento de riesgo con la politerapia (con antipsicóticos de segunda generación y con combinaciones de fármacos), los criterios diagnósticos actuales y el seguimiento de pacientes con riesgo de desarrollarlo. Pretendemos contribuir a una detección temprana de este síndrome que facilite implementar intervenciones multidisciplinarias farmacológicas y no farmacológicas.

PALABRAS CLAVE: Antipsicóticos. Síndrome Metabólico. Enfermedad Cardiovascular. Resistencia a la Insulina. Obesidad.
ABSTRACT:Although some second generation antipsychotic agents reduce the risk of extrapyramidal adverse effects against first generation agents, they have been associated to an increased incidence of metabolic syndrome. This syndrome is characterized by insulin resistance, abdominal obesity, atherogenic dyslipidemia (hypertriglyceridemia, decrease of HDL-cholesterol/ LDL-cholesterol ratio), arterial hypertension and a prothrombotic and proinflammatory state. Metabolic syndrome increases significantly the risk of cardiovascular illness and premature death. Therefore, a multidisciplinary approach to that syndorme's prevention and treatment could result in a substantially impact upon the general state of patients meetings its criteria. We emphasize current physiopathological findings, increased risk with polytherapy (second generation antipsychotics and both second and first generation combination), current diagnostic criteria and monitoring of patients at risk. Our aim is to contribute to an early detection that possibilities implementation of pharmacological and non pharmacological multidisciplinary interventions.

KEY WORDS: Antipsychotic Agents. Metabolic Syndrome. Cardiovascular Disease. Insulin Resistance. Obesity.

\section{AGRADECIMIENTOS}

Quiero expresar mi agradecimiento al Dr. Vicente Molina Rodríguez por sus comentarios y sugerencias al borrador de este manuscrito.

\section{CONFLICTO DE INTERESES:}

El autor declara no tener ningún conflicto de intereses. 


\section{Introducción}

Los antipsicóticos de segunda generación (AP2G), como grupo, son considerados fármacos que reducen el riesgo de producir efectos adversos de tipo extrapiramidal e hiperprolactinemia si son comparados con los antipsicóticos de primera generación (AP1G) (1,2). Sin embargo, hay evidencia que algunos de estos últimos aumentan el riesgo de un aumento de peso significativo, resistencia a la insulina (conocida como la disminución de la función biológica de la insulina caracterizada por requerir un alto nivel de insulina plasmática para mantener la homeostasis metabólica), hiperglucemia, diabetes mellitus (DM) tipo 2 y dislipidemia $(3,4,5,6)$, lo que podría reducir la expectativa de vida en pacientes que necesiten estos fármacos.

Los pacientes con enfermedades mentales graves como la esquizofrenia y el trastorno bipolar tienen un substancial aumento del riesgo de muerte comparado con la población general. En Estados Unidos, se ha calculado que la media años potencialmente perdidos en pacientes con enfermedad mental va de 25 a 30 , comparada con la población general (7). En este mismo estudio, la principal causa de muerte fue la enfermedad coronaria, y aunque la tasa de muerte es similar a la tasa en la población general, esta ocurría a edades más tempranas.

La aparición de aumento de peso, resistencia a la insulina (RI), hiperglicemia, DM tipo 2 y dislipidemia forman parte de lo que se ha denominado Síndrome Metabólico (SMet), Síndrome de resistencia a la insulina o Síndrome X, una entidad que se está convirtiendo en unos de los principales problemas de salud pública del siglo XXI, pues ha demostrado aumentar en 3 veces la prevalencia de la enfermedad cardiovascular en la población general (8).

Debido a que se ha encontrado una mayor presencia de SMet en pacientes con esquizofrenia que aun no habían iniciado tratamiento en comparación con la población general (9) existe todavía el debate de la génesis del trastorno metabólico en relación a la posibilidad que pueda haber un sustrato común entre el SMet y la esquizofrenia, que la psicosis predisponga a una alteración endocrina por un mecanismo no conocido (10), una acción de los AP2G sobre el metabolismo, o simplemente porque los pacientes haya tenido un estilo de vida no saludable antes de manifestar la psicosis.

En el presente artículo, se describirán las características de esta patología, la relación que tiene con el uso de antipsicóticos, y de qué manera se puede prevenir y detectar para un adecuado abordaje médico y dietético. 
ORIGINALES Y REVISIONES

¿Qué es el síndrome metabólico?

El SMet es una identidad descrita en 1923 por el médico sueco Eskil Kylin, quién definió la asociación entre hipertensión, hiperglucemia y gota (11). En un artículo sobre SMet, Zimmet y cols. (12), citan al médico español Gregorio Marañón quién señalaba que "la hipertensión arterial es un estado prediabético [...] este concepto también se aplica a la obesidad [...] y debe haber alguna forma de predisposición de carácter general para la asociación de la diabetes (del adulto) con la hipertensión arterial, la obesidad y quizá también con la gota [...] de manera que la dieta es esencial para la prevención y el tratamiento de todas estas alteraciones". Desde esa época hasta los años 80’s ha habido más descripciones de esta entidad. En 1988, Raven (13) lo describió como "Síndrome X" que incluía un conjunto de alteraciones metabólicas que tenían como centro la RI, aunque este no describió la obesidad como una característica del síndrome.

Sin profundizar en estos momentos en los criterios diagnósticos, el SMet se puede definir como la conjunción de factores de riesgo cardiometabólicos asociados a la RI, los cuales son: obesidad abdominal (excesivo tejido adiposo en y alrededor del abdomen), dislipidemia aterogénica (hipertrigliceridema, disminución del colesterol-HDL y aumento del colesterol-LDL), hipertensión arterial, intolerancia a la glucosa, estado protrombótico (elevación en sangre del fibrinógeno o inhibidor del activador del plasminógeno tipo 1) y estado proinflamatorio (elevación en sangre de la proteína C reactiva) (14). Estos factores de riesgo favorecen el desarrollo de enfermedades cardiovasculares, que incluyen enfermedad coronaria y cerebrovascular, así como DM tipo 2 (6).

\section{¿Cuál es su frecuencia?}

El SMet es muy frecuente en pacientes con enfermedades mentales graves. Se ha encontrado la presencia de SMet en pacientes con esquizofrenia que aún no habían iniciado tratamiento o en los que solo los habían tomado por dos semanas (9).

Los datos de prevalencia varían según los sujetos y los criterios clínicos de clasificación. La prevalencia en pacientes crónicos diagnosticados de esquizofrenia en una muestra de 240 sujetos canadienses mostró cifras de 42,6\% para hombres y 48,5\% para mujeres utilizando los criterios de la National Colesterol Education Program ATP (ATP III) (15). Los datos generados por el estudio CATIE (Clinical Antipsychotic Trials of Intervention Effectiveness) (16) mostraron una prevalencia general del 40,9\% con los criterios de la ATP III, siendo de 36,6\% para hombres y $54,2 \%$ para mujeres. En otro estudio realizado con 430 pacientes esquizofrénicos 
crónicos en Bélgica (17), la presencia de SMet alcanzaba prevalencias del 28,4\%, y $36 \%$, de acuerdo a los criterios diagnósticos de la ATP III y de la International Diabetes Federation, respectivamente, siendo más prevalente en pacientes femeninas.

En Suecia, Hägg y cols. (18), realizaron un estudio con 269 pacientes esquizofrénicos crónicos utilizando los criterios de la ATP III, encontrando una prevalencia del 34,6\%, siendo muy similares para hombres y mujeres. En España, se encontró una prevalencia de $36 \%$ en una muestra de 136 pacientes ingresados en el Hospital Universitario de Gran Canaria utilizando los criterios de la ATP III (19). En latinoamérica, un estudio realizado con pacientes ingresados en el Hospital Governador Israel Pinheiro de Brazil encontró prevalencias de 29,4\% en una muestra de 170 pacientes ingresados, siendo mayor en mujeres que en hombres $(43,6 \%$ y 20,8\%, respectivamente), utilizando también los criterios de la ATP III (20).

\section{¿Cómo se diagnostica?}

En 1998, la Organización Mundial de la Salud (WHO, por sus siglas en inglés) desarrolló su definición teniendo como criterio indispensable la RI (evidenciada por una aumento de los niveles de glucosa en ayunas, test de sobrecarga oral de glucosa alterado o un valor elevado un indicador de resistencia a la insulina como el HOMA-IR) (21)*.1 Además de este criterio, para el diagnóstico era necesario cumplir dos criterios adicionales que podían incluir obesidad, dislipidemia, hipertensión y microalbuminuria. La definición de la WHO fue la primera en enlazar los componentes claves de la RI, la obesidad, la dislipidemia y la hipertensión. Esta definición permitió también diagnosticar SMet en pacientes con DM tipo 2 que cumplían los otros criterios.

En 1999, el Grupo Europeo para el Estudio de la RI (EGIR, por sus siglas en inglés) propuso una modificación a los criterios diagnósticos de la WHO (22). En esta se simplificaba el criterio de obesidad midiendo la circunferencia de la cintura y no el índice cadera/cintura o al índice de masa corporal (IMC) y excluyó la presencia de microalbuminuria como criterio diagnóstico. La RI es también central en la definición, aunque no permite que un paciente diagnosticado con DM tipo 2 sea también diagnosticado con este síndrome.

En el año 2002, el NCEP ATP III (National Cholesterol Education Program Adult Treatment Panel III) (23) propuso una clasificación que fue actualizada pos-

HOMA-IR: Homeostatic model assessment of insulin resistance 
Tabla 1

Criterios diagnósticos del Síndrome Metabólico.

\begin{tabular}{|c|c|c|c|c|}
\hline & $\begin{array}{l}\mathrm{WHO}^{21} \\
(1998)\end{array}$ & $\begin{array}{l}\text { EGIR }^{22} \\
(1999)\end{array}$ & $\begin{array}{l}\text { NCEP ATP III }{ }^{23} \\
\text { (Revisión 2005) }\end{array}$ & $\begin{array}{l}\mathrm{IDF}^{25,26} \\
(2005)\end{array}$ \\
\hline $\begin{array}{l}\text { Presencia } \\
\text { indispensable de }\end{array}$ & RI o DM tipo 2 & $\begin{array}{l}\text { Hiperinsulinemia * } \\
\text { (insulina plasmática > } \\
\text { percentil 75) }\end{array}$ & & $\begin{array}{l}\text { Obesidad central: } \geq 94 \\
\mathrm{~cm} \text { en hombres } \mathrm{y} \geq 80 \\
\mathrm{~cm} \text { en mujeres } \dagger\end{array}$ \\
\hline CRITERIOS & $\begin{array}{l}\text { RI o DM más } 2 \text { de los } \\
5 \text { criterios siguientes }\end{array}$ & $\begin{array}{l}\text { Hiperinsulinemia más } \\
2 \text { de los } 4 \text { criterios } \\
\text { siguientes }\end{array}$ & $\begin{array}{l}\text { Cumplir al menos tres } \\
\text { de los siguientes }\end{array}$ & $\begin{array}{l}\text { Obesidad central más } \\
2 \text { de los } 4 \text { criterios } \\
\text { siguientes }\end{array}$ \\
\hline Obesidad & $\begin{array}{l}\text { Índice cadera/cintura: } \\
>0.90 \text { en hombres, } \\
0.85 \text { en mujeres; o } \\
\mathrm{IMC}>30 \mathrm{Kg} / \mathrm{m}^{2}\end{array}$ & $\begin{array}{l}\text { Circunferencia de } \\
\text { cintura: }>94 \mathrm{~cm} \text { para } \\
\text { hombres y } 80 \mathrm{~cm} \text { para } \\
\text { mujeres }\end{array}$ & $\begin{array}{l}\text { Circunferencia de } \\
\text { cintura: }>102 \mathrm{~cm} \text { para } \\
\text { hombres y } 88 \mathrm{~cm} \text { para } \\
\text { mujeres }\end{array}$ & Obesidad central \\
\hline Hiperglicemia & RI o DM tipo 2 & RI & $\begin{array}{l}\text { Hiperglicemia } \geq 100 \\
\mathrm{mg} / \mathrm{dl} \text { o presencia de } \\
\text { algún tratamiento } \\
\text { específico para su } \\
\text { control. }\end{array}$ & $\begin{array}{l}\text { Hiperglicemia } \geq 100 \\
\mathrm{mg} / \mathrm{dL}\end{array}$ \\
\hline Dislipidemia (1) & $\begin{array}{l}\text { Trigliceridemia } \geq 150 \\
\mathrm{mg} / \mathrm{dl} \text { o Colesterol- } \\
\mathrm{HDL}<35 \mathrm{mg} / \mathrm{dL} \text { para } \\
\text { hombres } \mathrm{y}<39 \mathrm{mg} / \mathrm{dL} \\
\text { para mujeres }\end{array}$ & $\begin{array}{l}\text { Trigliceridemia } \geq 177 \\
\mathrm{mg} / \mathrm{dl} \text { o Colesterol- } \\
\mathrm{HDL}<39 \mathrm{mg} / \mathrm{dL}\end{array}$ & $\begin{array}{l}\text { Hipertrigliceridemia } \\
\geq 150 \mathrm{mg} / \mathrm{dL} \text { o } \\
\text { presencia de algún } \\
\text { tratamiento específico } \\
\text { para su control. }\end{array}$ & $\begin{array}{l}\text { Trigliceridemia } \geq 150 \\
\text { mg/dl o presencia de } \\
\text { algún tratamiento } \\
\text { específico para su } \\
\text { control. }\end{array}$ \\
\hline Dislipidemia (2) & & & $\begin{array}{l}\text { Colesterol-HDL }<40 \\
\mathrm{mg} / \mathrm{dl} \text { en hombres o }< \\
50 \mathrm{mg} / \mathrm{dL} \text { en mujeres, } \\
\text { o presencia de algún } \\
\text { tratamiento específico } \\
\text { para su control. }\end{array}$ & $\begin{array}{l}\text { Colesterol-HDL }<40 \\
\mathrm{mg} / \mathrm{dl} \text { en hombres o }< \\
50 \mathrm{mg} / \mathrm{dL} \text { en mujeres, } \\
\text { o presencia de algún } \\
\text { tratamiento específico } \\
\text { para su control. }\end{array}$ \\
\hline Hipertensión arterial & $\geq 140 / 90 \mathrm{mmHg}$ & $\geq 140 / 90 \mathrm{mmHg}$ & $\begin{array}{l}\text { PAS }>130 \mathrm{mmHg} \text { o } \\
\text { PAD }>85 \mathrm{mmHg} \text { o } \\
\text { presencia de algún } \\
\text { tratamiento específico } \\
\text { para su control. }\end{array}$ & $\begin{array}{l}\text { PAS }>130 \mathrm{mmHg} \text { o } \\
\text { PAD }>85 \mathrm{mmHg} \text {, o } \\
\text { presencia de algún } \\
\text { tratamiento específico } \\
\text { para su control. }\end{array}$ \\
\hline Otro criterios & Microalbuminuria $\ddagger$ & & & \\
\hline
\end{tabular}

* Solo para pacientes sin DM tipo 2

${ }^{\dagger}$ Los valores de referencia para el diagnóstico de obesidad central son específicos para cada población. 25

‡ Excreción urinaria de albúmina $\geq 20 \mu \mathrm{g} / \mathrm{min}$ o relación albúmina/creatinina $\geq 30 \mathrm{mg} / \mathrm{g}$

WHO: World Health Organization, EGIR: European Group for the Study of Insulin Resistance, NCEP ATP III: Expert Panel on Detection, Evaluation, and Treatment of High Blood Cholesterol in Adults (Adult Treatment Panel III), IDF: International Diabetes Foundation, RI: resistencia a la insulina, DM: diabetes mellitus, HDL: high density lipoprotein, PAS: presión arterial sistólica, PAD: Presión arterial diastólica. 
teriormente en el año 2005 por la AHA (American Heart Asociation) y el NHLBI (National Heart Lung and Blood Institute) (24). En esta clasificación denominada NCEP ATP III, no es un criterio indispensable la RI, pues no fue concebida por los autores como la causa subyacente del SMet. Para hacer el diagnóstico de SMet según estos criterios, es necesario cumplir solamente tres criterios de una lista de cinco, que incluye entre estos los niveles de colesterol-HDL y no solo los niveles de triglicéridos.

Finalmente, en ese mismo año, la IDF (International Diabetes Foundation) publicó unos criterios diagnósticos que incluía a todos los componentes de la clasificación $(25,26)$, e igualmente no incluía la presencia necesaria de la RI. Una diferencia relevante en relación a las anteriores clasificaciones es que la circunferencia de la cintura fue especificada por zonas geográficas, tomando en cuenta la antropometría local.

En la actualidad la mayoría de las investigaciones utilizan los criterios de la NCEP ATP III y de la IDF. La tabla 1 resume los criterios anteriormente mencionados.

¿Cómo se explica fisiopatológicamente?

Stahl \& Meyer $(1,27,28)$, afirman que aún no se conocen con claridad los mecanismos por los cuales algunos de los AP2G pueden producir SMet. Estos autores resumen la fisiopatología como una cadena de acontecimientos que terminan produciendo una situación de riesgo cardiovascular.

El aumento de peso, resistencia a la insulina e hiperinsulinemia.

El incremento del apetito provocado por un lado, por el bloqueo de los receptores histaminérgicos $\mathrm{H} 1$ en los centros del hambre en el hipotálamo y por el bloqueo serotoninérgico 5-HT2C por otro, explica la tendencia a la obesidad por los AP2G $(27,29)$. Teórica y prácticamente no todos los antipsicóticos bloquean los mismos receptores ni tampoco en igual medida, por lo que aquellos fármacos que producen un mayor aumento de peso son aquellos que producen mayor antagonismo en estos receptores (1), ver tabla 2.

El aumento de peso, específicamente la obesidad central, se relaciona fuertemente con la RI (9). De forma fisiológica, la homeostasis de la glucosa en sangre es modulada por la insulina, el glucagón y otras sustancias como aminoácidos, 
ORIGINALES Y REVISIONES

Tabla 2

Antipsicóticos de segunda generación y riesgo de aumento de peso (1)

\begin{tabular}{|c|c|}
\hline Antipsicótico & Riesgo de aumento de peso \\
\hline Clozapina & +++ \\
\hline Olanzapina & +++ \\
\hline Risperidona & ++ \\
\hline Quetiapina & ++ \\
\hline Ziprasidona & ++- \\
\hline Aripiprazol & $+/-$ \\
\hline
\end{tabular}

cetonas, péptidos gastrointestinales y neurotransmisores (30). Las concentraciones de glucosa que sobrepasan los $70 \mathrm{mg} / \mathrm{dL}$ estimulan la síntesis de insulina por las células beta localizadas en los islotes de Langerhans del páncreas, para mantener la homeostasis de este sustrato en la sangre (30). La glucosa comienza a estimular la secreción de insulina cuando aquella es introducida en la célula beta por el transportador de glucosa (30). Una vez secretada la insulina, esta pasa hacia el sistema venoso portal y casi el $50 \%$ de ella es degradada por el hígado (30). La insulina que no es metabolizada llega a la circulación general, donde se fija a los receptores de membrana en las células de sus órganos diana (hígado, músculo esquelético y tejido adiposo) (30), lo que a su vez produce una cascada de segundos mensajeros intracelulares que termina por introducir la glucosa en la célula y por consecuencia la disminución de la concentración de glucosa en sangre (30)

La mayoría de los investigadores dan un lugar prioritario a la RI frente a la obesidad como el principal factor en la patogénesis del síndrome metabólico (31). La RI que acompaña a la obesidad parece deberse a la producción por el adipocito de cierto número de productos biológicos como la leptina, ácidos grasos libres, adiponeptina y otras sustancias (factor de necrosis tumoral alfa y resistina) que modulan entre otras cosas la secreción de insulina y la sensibilidad a la misma, de tal manera que un aumento en la producción de estas sustancias demandan un aumento en la secreción de esta hormona (30).

Actualmente existe evidencia que sugiere que el uso crónico de algunos AP2G incrementan los niveles de leptina como producto de la obesidad produciendo un aumento de peso (32). Sin embargo Hagg y cols. (33), describieron pacientes en los que los niveles de leptina estaban aumentados independientemente del peso en pacientes tratados con clozapina.

La hiperinsulinemia, como producto de la secreción de los productos sintetizados por el adiposito, produce una menor sensibilidad de sus receptores en los órganos diana y la disminución de la utilización de glucosa en esas células, de tal forma que se requieren concentraciones de insulina superiores a lo normal para producir una respuesta cuantitativamente adecuada (27). La disminución de la 
utilización de glucosa produce un aumento de su concentración en sangre que demanda aún más la secreción de insulina. En fases tempranas la glucosa se mantiene normal porque las células beta compensan aumentando la producción de insulina. A medida que avanza la RI las células beta se tornan incapaces de mantener el estado de hiperinsulinismo hasta llegar a un fallo aún mayor originando la DM tipo 2 (27). Paralelamente a esta vía, se ha propuesto que las concentraciones elevadas de ácidos grasos libres, aspecto frecuente en la obesidad, pueden obstaculizar el empleo de la glucosa por el músculo esquelético, promover la producción de este azúcar por el hígado y trastornar la función de la célula beta debido a que también demanda el aumento de la secreción de insulina (27) (figura 1).

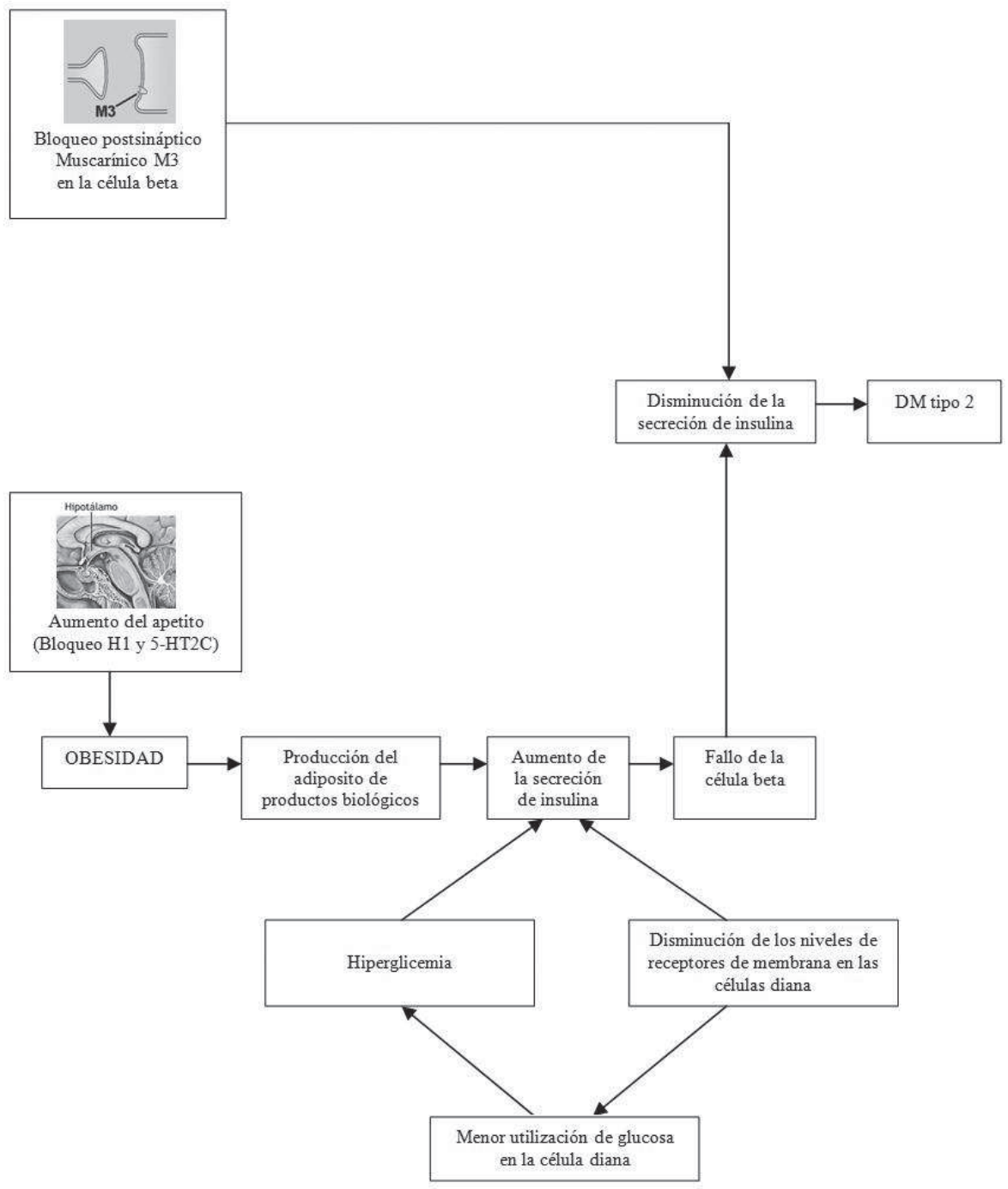

Figura 1. Esquema de la cascada de acontecimientos propuestos para explicar la resistencia a la insulina y la diabetes mellitus tipo 2 asociada a los antipsicóticos de segunda generación. 
Otro mecanismo propuesto para explicar la RI por AP2G es el antagonismo muscarínico M3, como el producido por la olanzapina y la clozapina (27). Los receptores M3 postsinápticos están localizados en la célula beta, de esta manera, el bloqueo de estos receptores puede conducir a una disminución directa de la secreción de la insulina (27).

Los AP2G han sido asociados también a otras raras pero peligrosas complicaciones agudas de la DM tipo 2, la cetoacidosis diabética (28) y el síndrome hiperosmolar (10). Se presume que pacientes con una RI, prediabetes o DM no sintomática, aun no diagnosticada a consecuencia de la compensación de unas células beta pancráticas aun normofuncionantes, pueden desarrollar estas complicaciones debido a la introducción del AP2G y el consecuente fallo de estas células por los mecanismos antes explicados.

\section{Hipertrigliceridemia.}

La RI produce también hipertrigliceridemia debido a que las lipasas dependientes de insulina en las células grasas son normalmente inhibidas por esta hormona (28). La falta de función de la insulina en los adipositos produce elevados niveles de lipólisis y la liberación de un exceso de ácidos grasos del tejido adiposo que en el hígado son transformados en triglicéridos (28). El exceso de ácidos grasos libres predispone al depósito de estos en otros tejidos de la economía. La progresiva acumulación de ácidos grasos e hiperglucemia podría originar un fenómeno de toxicidad en la célula beta (34) (glucolipotoxicidad) que también son determinantes en el agotamiento de la capacidad secretora de la célula beta.

La hipertrigliceridemia se explica también por la alteración de la función reguladora de la apolipoproteina B100 (ApoB100) (28). Las apolipoproteínas son proteínas en la superficie del complejo de lipoproteínas que se enlazan a enzimas específicas o transportan proteínas en las membranas celulares (35). La ApoB100 es esencial para la producción intracelular de VDRL (que son las lipoprotenínas con mayor cantidad de triglicéridos) (36). La sobreproducción de ApoB100 por la lipólisis ya comentada, genera más cantidad de partículas ricas en triglicéridos con la consecuente hipertrigliceridemia (28).

\section{Disminución de los niveles de HDL.}

La elevada presencia de las lipoproteínas ricas en triglicéridos causa la transferencia de triglicéridos a lipoproteínas de alta densidad (HDL) a expensas del 
colesterol-HDL (28). Después del paso de las HDL a través del hígado, donde los triglicéridos son divididos por procesos enzimáticos, las restantes partículas de HDL son más pequeñas de lo normal y más fácilmente aclaradas por el riñón, produciendo los bajos niveles de HDL observados en los estados de RI (28).

Hipótesis de un receptor " $X$ ”.

Según Stahl, Mignon y Meyer $(1,27)$, hay evidencia que antipsicóticos como la clozapina y la olanzapina, elevan los niveles de triglicéridos e incrementan la RI de una forma que no puede ser explicada por la inhibición de las lipasas dependientes de insulina, ya que se ha observado un rápido aumento de los triglicéridos al iniciar el tratamiento y un rápido descenso al suspenderlo (27). Debido a la falta de una explicación fisiopatológica, se ha hipotetizado la presencia de un "receptor X" todavía no identificado en el tejido adiposo, hígado, músculo esquelético y posiblemente en el cerebro, que puede ser bloqueado por el AP2G (27). Esto podría explicar porqué estos fármacos elevan rápidamente los niveles de triglicéridos y su disminución tras la discontinuación (27).

\section{Estado proinflamatorio y protrombótico.}

Aunque todavía la fisiopatología de estos estados es incierta se cree que son múltiples los mecanismos subyacentes. Una de las causas puede ser la misma obesidad, porque el exceso de tejido adiposo libera citoquinas inflamatorias que pueden elevar los niveles de proteína $\mathrm{C}$ reactiva (PCR) y de fibrinógeno (31). Se ha encontrado también una elevación del inhibidor del activador de plasminógeno tipo 1 (PAI-1) (31).

¿Es el uso de antipsicóticos de segunda generación un factor de riesgo independiente de otras variables clínicas?

La presencia de SMet relacionado con el tratamiento de AP2G debe también valorarse a la luz de variables como la posible predisposición genética del paciente, estilos de vida (dieta, sedentarismo y tabaquismo), y variables socio-demográficas como la pobreza, acceso a la atención médica, falta de un seguro médico y la ur- 
ORIGINALES Y REVISIONES

banización (37). Además, a diferencia de otros, los pacientes con esquizofrenia no suelen acudir al médico de cabecera, al cardiólogo o al endocrinólogo ante el deterioro de su salud.

Sin embargo, hay datos a favor que los AP2G son un factor de riesgo (FR) independiente a estas otras variables. En una publicación reciente (27), se presentaron los resultados de una revisión que se proponía resolver esta controversia y se encontró fuerte evidencia del riesgo cardiometabólico entre los diferentes AP2G, debido al antagonismo $\mathrm{H} 1$ y $5 \mathrm{HT} 2 \mathrm{C}$ que estadísticamente estaba asociado al riesgo del aumento de peso y la hipertrigliceridemia. Los autores concluyen que, aunque el estilo de vida, la carga genética pueden contribuir de forma independiente al riesgo cardiometabólico en la esquizofrenia y en otras enfermedades mentales graves, el uso de AP2G puede incrementarlo.

La cuestión se magnifica cuando se utilizan asociaciones de antipsicóticos. Se sabe que alrededor del 15\% de los pacientes ambulatorios y del 50\% de los hospitalizados en Estados Unidos reciben dos o más antipsicóticos (38), cifra que alcanza el 35,6\% en un estudio noruego (39) y el 90\% en otros países como Japón (38).

Para determinar si la prescripción de dos o más antipsicóticos estaba asociado a un incremento del riesgo de desarrollar el desorden metabólico, se realizó un estudio en 367 adultos tratados con más de un AP2G y diagnosticados con SMet $(40,41)$. La prevalencia de politerapia antipsicótica alcanzaba el 19,2\%, y fue significativamente mayor para aquellos pacientes tratados con clozapina, quetiapina o ziprasidona. En comparación con la monoterapia, la politerapia se asoció con unas tasas más elevadas de SMet $(50,0 \%$ vs. $34,3 \%, \mathrm{p}=0,015)$ y del índice TG/HDL (50,7\% vs. $35,0 \%, p=0,016)$. Sin embargo, en el análisis de regresión logística, el SMet fue asociado significativamente también a un elevado IMC, edad avanzada, diagnóstico de trastorno bipolar o esquizofrenia, y asociación con un AP1G. Concluyeron que comparado con la monoterapia, la politerapia antipsicótica tiene más altas tasas de SMet, sin embargo, esta no es independiente con la prevalencia de los factores demográficos, clínicos y antropométricos.

\section{¿Qué hacer al respecto?}

Los psiquiatras deben aprender a reconocer los signos que alertan de problemas cardiometabólicos relacionados a los antipsicóticos y así prevenir la progresión a DM tipo 2, enfermedades cardiovasculares y muerte prematura. 


\section{Control metabólico.}

El seguimiento metabólico ha sido reiterado varias veces a través de consensos y guías clínicas. La mayoría de estas afirman que el psiquiatra y otros médicos relacionados con los servicios de salud mental deberían obtener datos basales del paciente y luego monitorizarlos a lo largo del tratamiento (37).

Son cuatro los parámetros que deben registrarse en estos pacientes en relación al SMet:

- Tensión Arterial

- Peso, IMC

- Glicemia

- Perfil lipídico (colesterol-total, colesterol-LDL, colesterol-HDL y triglicéridos)

En este artículo se describe el seguimiento de los parámetros publicados por el Consenso sobre la salud física del paciente con esquizofrenia de las Sociedades Españolas de Psiquiatría y Psiquiatría Biológica (tabla 3) (42). Sin embargo, se pueden consultar otras guías como la publicada por la Asociación Americana de Psiquiatría (43), y el Grupo de Trabajo sobre Esquizofrenia de la Asociación Canadiense de Psiquiatría (44).

Tabla 3

Evaluación de parámetros clínicos y de laboratorio (42)

\begin{tabular}{|c|c|c|}
\hline Variables & Basal & Seguimiento \\
\hline Signos vitales & $\begin{array}{l}\text { Frecuencia cardíaca y } \\
\text { tensión arterioal }\end{array}$ & A los $6,12,18$ y 24 meses \\
\hline Peso & Peso e IMC & A los $6,12,18$ y 24 meses. \\
\hline Glicemia, Lípidos & $\begin{array}{l}\text { Búsqueda de factores de riego para DM, } \\
\text { glicemia en ayunas. Perfil lipídico }\end{array}$ & $\begin{array}{l}\text { A los } 12 \text { y } 24 \text { meses. Excepto si: } \\
\text { - Glucemia > } 125 \mathrm{mg} / \mathrm{dL} \text {, repetir en la } \\
\text { siguiente visita } \\
\text { - Cambio de tratamiento antipsicótico, } \\
\text { repetir a las } 10 \text { semanas y a los } 6 \text { meses } \\
\text { - Repetir si hay incremento de peso }>5 \%\end{array}$ \\
\hline
\end{tabular}

IMC: índice de masa corporal, DM: diabetes mellitus, Hemoglobina A1c: hemoglobina glicosilada

El IMC se puede calcular usando la formula IMC = Peso en $\mathrm{kg} /$ talla en metros al cuadrado, o usando las tablas disponibles por el National Institute of Diabetes and Digestive and Kidney Disease (http://win.niddk.nih.gov/statistics/\#table). Como alternativa al IMC también puede utilizarse la circunferencia de cintura medida a la altura del ombligo. El peso suele medirse cada seis meses o al menos de forma trimestral, excepto en pacientes con IMC $<18.5$. Un incremento del IMC en 
ORIGINALES Y REVISIONES

una unidad debería sugerir la necesidad de monitorizar el peso con más frecuencia, introduciendo al paciente en un programa para el control de peso.

Se deben buscar FR para DM $(30,44)$ como un IMC $>25$, la presencia familiares de primer grado diagnosticados de diabetes, el sedentarismo habitual, el pertenecer a poblaciones étnicas de riesgo (raza negra, hispanoamericanos, nativos norteamericanos, asiáticos y de las íslas del pacífico), haber dado a luz un bebé macrosómico $>4000 \mathrm{~g}$ o haber presentado diabetes gestacional, hipertensión, niveles de colesterol-HDL $<35 \mathrm{mg} / \mathrm{dL}$ o triglicéridos $>250 \mathrm{mg} / \mathrm{dL}$, una historia de alteraciones en el test de tolerancia oral a la glucosa o glicemia en ayunas, síndrome de ovario poliquístico o acantosis nigricans, e historia de enfermedad vascular.

Como una complemento a la toma de glicemia en ayunas, se deben tomar niveles de Hemoglobina A1c. Valores anormales (glicemia $>110 \mathrm{mg} / \mathrm{dl}$ o hemoglobina A1c $>6.1 \%$ ) indican la remisión del paciente a consulta especializada. Si el hallazgo de una glicemia $>200 \mathrm{mg} / \mathrm{dl}$ en cualquier hora del día, se debe realizar seguimiento periódico más frecuente en relación a los cambios de peso e indagar sobre síntomas de diabetes (poliuria, polidipsia, polifagia, aumento de peso, fatiga, irritabilidad y visión borrosa).

\section{Intervenciones conductuales y cambios en la medicación.}

Una importante conclusión del programa de prevención de la diabetes es que una intervención intensiva en el estilo de vida de la persona es la mejor medida para mejorar la sensibilidad a la insulina y reducir la incidencia futura de DM tipo 2. Los pacientes deben ser alentados a dejar de fumar y disminuir el peso ofreciéndoles alternativas psicoterapéuticas y/o farmacológicas para tales fines.

$\mathrm{El}$ aumento de peso asociado a los AP2G generalmente ocurre en los primeros meses del inicio del tratamiento y puede no estabilizarse hasta más de un año (45). Es recomendable también reducir el consumo de grasas saturadas e incrementar el consumo de fibra y de actividad física (23).

Sin embargo, el factor de riesgo en el que puede incidir principalmente el médico especialista en psiquiatría es la prescripción del antipsicótico, dentro del marco clínico riesgo-beneficio. En la elección debe primar la eficacia del antipsicótico y la presencia de anormalidades metabólicas. En el caso de un episodio grave o una psicosis resistente al tratamiento, es mejor optimizar los fármacos eficaces en este paciente dejando en un segundo plano el riesgo metabólico, pues la psicosis grave afecta en mayor medida el funcionamiento individual, familiar y social que la aparición de SMet. En los casos, al aparecer anormalidades metabólicas, se debe tener en consideración cambiar a otro AP2G no asociado a elevado aumento de peso o 
aparición de diabetes (figura 2). La American Diabetes Asociation sugiere que, un aumento de peso mayor o igual al 5\% de la línea basal puede requerir cambio a otro antipsicótico (45).

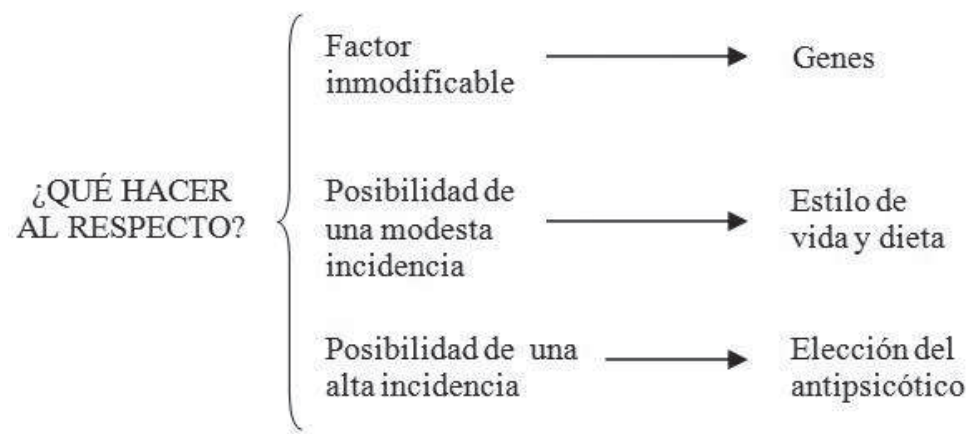

Figura 2. Varios factores influyen en el desarrollo de la RI. La edad del paciente y los genes son factores de riesgo inmodificables, mientras que el estilo de vida y la dieta pueden ser mejorados por la educación en salud. La mayor influencia en el riesgo CV por parte del psiquiatra es la elección una medicación antipsicótica apropiada.

De forma general general existen diferencias sustanciales en cuanto al perfil de efectos adversos entre los AP2G. La risperidona y la paliperidona se asocian a mayor producción de síntomas extrapiramidales e hiperprolactinemia; la olanzapina y la clozapina, seguida de la quetiapina, se asocian a una mayor presentación de alteraciones metabólicas; con el aripiprazol y la ziprasidona no se detectan estas últimas alteraciones, no obstante el primero se asocia a acatisia y el segundo a prologación del segmento QT en el electrocardiograma. A manera de resumen de los efectos metabólicos de los AP2G se presenta la revisión hecha por Tamminga y cols. (46), los cuales puntúan la asociación de SMet con cada uno de estos fárma$\cos ($ tabla 4$)$

Tabla 4

Antipsicóticos de segunda generación y su asociación con la aparición de síndrome metabólico (46)

\begin{tabular}{|c|c|}
\hline Fármaco & Síndrome metabólico \\
\hline Clozapina & +++ \\
\hline Olanzapina & $++/+++$ \\
\hline Quetiapina & ++ \\
\hline Risperidona & + \\
\hline Ziprasidona & $0 /+$ \\
\hline Aripiprazol & $0 /+$ \\
\hline
\end{tabular}


ORIGINALES Y REVISIONES

\section{Conclusiones.}

La presencia de SMet y otros factores de riesgo cardiovasculares tienen alta prevalencia en pacientes con enfermedad mental grave. El tratamiento con AP2G es esencial en una gran cantidad de casos, y al usarlos se debe tener presente que algunos de estos se consideran una de las variables que influyen en la aparición del SMet mediante una cadena de mecanismos que van desde el aumento del apetito y consecuente aumento de peso, hasta el desarrollo de una resistencia a la insulina y diabetes mellitus.

No se pretende que el psiquiatra sea un endocrinólogo o un especialista en medicina interna, sino que estos deben conocer este riesgo para incluir en el tratamiento una valoración del riesgo/beneficio del antipsicótico usado ya que también un incremento en la efectividad para reducir los síntomas valorado como superior a las reacciones adversas incrementan las oportunidades del paciente para llevar una vida sin menoscabo personal, familiar y social.

BIBLIOGRAFÍA:

(1) Stahl SM. Stahl's Essential Psychopharmacology: Neuroscientific Basis and Practical Applications. 3rd edition. New York: Cambridge University Press; 2008

(2) Panksepp J (editor). Textbook of biological psychiatry. New Jersey: Wiley-Liss, Inc.; 2004

(3) Allison DB, Mentore JL, Heo M, Chandler LP, Cappelleri JC, Infante MC, et al. Antipsychotic-induced weight gain: a comprehensive research synthersis. Am J Psychiatry. 1999;156(11):168696.

(4) McElroy SL. Obesity in patients with severe mental illness: overview and management. J Clin Psychiatry. 2009;70 Suppl 3:12-21.

(5) Buchholz S, Morrow AF, Coleman PL. Atypical antipsychotic-induced diabetes mellitus: an update on epidemiology and postulated mechanisms. Intern Med J. 2008 Jul;38(7):602-6.

(6) Newcomer, JW. Second-generation (atypical) antipsychotics and metabolic effects: a comprehensive literature review. CNS Drugs. 2005;19 Suppl 1:1-93.

(7) Colton C, Manderscheid R. Congruencies in increased mortality rates, years of potential life lost, and causes of death among public mental health clients in eight states. Prev Chronic Dis. 2006 Apr;3(2):A42. Epub 2006 Mar 15.

(8) Carmena R. Síndrome metabólico. En: Farreras P, Rozman C, (editores) Medicina Interna. Barcelona: Elservier; 2004. p. 1999-2000

(9) Patel JK, Buckley PF, Woolson S, Hamer RM, McEvoy JP, Perkins DO, et al. Metabolic profiles of second generation antipsychotics in early psychosis: findings from the CAFE study. Schizophr Res. 2009 Jun;111(1-3):9-16. Epub 2009 Apr 26.

(10) Seaburg HL, McLendon BM, Doraiswamy PM. Olanzapine-associated severe hyperglycemia, ketonuria, and acidosis: case report and review of literature. Pharmacotherapy. 2001 Nov;21(11):144854. 
(11) Kylin E. Studien uber das Hypertonie-Hyperglyka 'mie-Hyperurika' miesyndrom. Zentralbl Inn Med 1923;44:105-127.

(12) Zimmet P, Alberti KG, Serrano M. Una nueva definición mundial del síndrome metabólico propuesta por la Federación Internacional de Diabetes: fundamento y resultados. Rev Esp Cardiol. 2005;58(12):1371-6

(13) Raven G. Banting lecture 1988. Role of insulin resistance in human disease. Diabetes. 1988 Dec;37(12):1595-607.

(14) Metabolic syndrome [page on Internet]. Dallas: American Heart Association, Inc.; c2009 [updated 2009 Dec 16; cited 2009 Sep 12]. Available from http://www.americanheart.org/presenter. jhtml?identifier $=4756$

(15) Cohn T, Prud'Homme D, Streiner D, Kameh H, Remington G. Characterizing coronary heart disease risk in chronic schizophrenia: high prevalence of the metabolic syndrome. Can J Psychiatry. 2004 Nov;49(11):753-60.

(16) McEvoy JP, Meyer JM, Goff DC, Nasrallah HA, Davis SM, Sullivan L, et al. Prevalence of the metabolic syndrome in patients with schizophrenia: baseline results from the Clinical Antipsychotic Trials of Intervention Effectiveness (CATIE) schizophrenia trial and comparison with national estimates from NHANES III. Schizophr Res. 2005 Dec 1;80(1):19-32. Epub 2005 Aug 30.

(17) De Hert MA, van Winkel R, Van Eyck D, Hanssens L, Wampers M, Scheen A, et al. Prevalence of the metabolic syndrome in patients with schizophrenia treated with antipsychotic medication. Schizophr Res. 2006 Mar;83(1):87-93. Epub 2006 Feb 14.

(18) Hägg S, Lindblom Y, Mjörndal T, Adolfsson R. High prevalence of the metabolic syndrome among a Swedish cohort of patient with schizophrenia. Int Clin Psychopharmacol. 2006 Mar;21(2):93-8.

(19) Sánchez-Araña T, Touriño R, Hernández JL, León P. Prevalencia del síndrome metabólico en pacientes esquizofrénicos hospitalizados en Gran Canaria. Actas Esp Psiquiatr 2007;35(6):359-367

(20) Ribeiro PJ, Lopes F. The prevalence of metabolic syndrome among psychiatric inpatients in Brazil. Rev Bras Psiquiatr , 29 (4), 330-6.

(21) Alberti KG, Zimmet PZ. Definition, diagnosis and classification of diabetes mellitus and its complications. Part 1: diagnosis and classification of diabetes mellitus provisional report of a WHO consultation. Diabet Med. 1998 Jul;15(7):539-53.

(22) Balkau B, Charles MA. Comment on the provisional report from the WHO consultation. European Group for the Study of Insulin Resistance (EGIR). Diabet Med. 1999 May;16(5):442-3.

(23) National Cholesterol Education Program Expert Panel on Detection, Evaluation, and Treatment of High Blood Cholesterol in Adults. Third Report of the National Cholesterol Education Program (NCEP) Expert Panel on Detection, Evaluation, and Treatment of High Blood Cholesterol in Adults (Adult Treatment Panel III) final report. Circulation. 2002 Dec 17;106(25):3143-421.

(24) Grundy SM, Cleeman JI, Daniels SR, Donato KA, Eckel RH, Franklin BA, et al. Diagnosis and Management of the Metabolic Syndrome: An American Heart Association/National Heart, Lung, and Blood Institute Scientific Statement. Circulation 2005;112;2735-2752

(25) Zimmet P, Magliano D, Matsuzawa Y, Alberti G, Shaw J. The metabolic syndrome: a global public health problem and a new definition. J Atheroscler Thromb. 2005;12(6):295-300.

(26) The IDF consensus worldwide definition of the metabolic syndrome [Internet]. Brussels: International Diabetes Federation; 2009 [cited 2009 Dec 15]. Available from: http://www.idf.org/webdata/ docs/MetS_def_update2006.pdf 
ORIGINALES Y REVISIONES

(27) Stahl SM, Mignon L, Meyer JM. Which comes first: atypical antipsychotic treatment or cardiometabolic risk? Acta Psychiatr Scand. 2009 Mar;119(3):171-9.

(28) Meyer JM, Stahl SM. The metabolic syndrome and schizophrenia. Acta Psychiatr Scand. 2009 Jan;119(1):4-14.

(29) Liberty IF, Todder D, Umansky R, Harman-Boehm I. Atypical Antipsychotics and Diabetes Mellitus: An Association. Isr Med Assoc J. 2004 May;6(5):276-9.

(30) Powers A. Diabetes mellitus. En: Kasper DL, Braunwald E, Fauci AS, Hauser SL, Longo DL, Jameson JL. Harrison: Principios de Medicina Interna. Chile: McGraw Hill; 2005. p. 2367-97.

(31) Grundy SM, Brewer HB, Cleeman JI, Smith SC, Lenfant C. Definition of metabolic syndrome: report of the National Heart, Lung, and Blood Institute/American Heart Association conference on scientific issues related to definition. Circulation 2004;109;433-438

(32) Jin H, Meyer JM, Mudaliar S, Jeste DV. Impact of Atypical Antipsychotic Therapy on Leptin, Ghrelin, and Adiponectin. Schizophrenia research 2007;100(1-3),70-85.

(33) Hägg S, Söderberg S, Ahrén B, Olsson T, Mjörndal T. Leptin concentrations are increased in subjects treated with clozapine or conventional antipsychotics. J Clin Psychiatry. 2001 Nov;62(11):843-8.

(34) Vidal-Puig A, Carmena Rodríguez R. Obesidad y síndrome metabólico. In Farreras P, Rozman C. Medicina Interna. Barcelona: Elservier; 2009. p. 1993-2000.

(35) Mahley RW, Innerarity TL, Rall SC Jr, Weisgraber KH. Plasma lipoproteins: apolipoprotein structure and function. J Lipid Res. 1984 Dec 1;25(12):1277-94.

(36) Olofsson SO, Borèn J. Apolipoprotein B: a clinically important apolipoprotein which assembles atherogenic lipoproteins and promotes the development of atherosclerosis. J Intern Med. 2005 Nov;258(5):395-410.

(37) Newcomer JW. Metabolic syndrome and mental illnes. Am J Manag Care. 2007;13:S170S177

(38) Villagrán JM, Luque R. Asociaciones de antipsicóticos en la práctica clínica: una revisión crítica. Barcelona: Prous Science; 2007.

(39) Kroken RA, Johnsen E, Ruud T, Wentzel-Larsen T, Jørgensen HA. Treatment of schizophrenia with antipsychotics in Norwegian. BMC Psychiatry. 2009 May 16;9:24.

(40) Correll CU, Frederickson AM, Kane JM, Manu P. Does Antipsychotic Polypharmacy Increase the Risk for Metabolic Syndrome? Schizophr Res. 2007 Jan;89(1-3):91-100. Epub 2006 Oct 27.

(41) Correll CU, Frederickson AM, Kane JM, Manu P. Metabolic syndrome and the risk of coronary heart disease in 367 patients treated with second-generation antipsychotic drugs. J Clin Psychiatry. 2006 Apr;67(4):575-83.

(42) Sáiz J, Bobes J, Ruiloba V, Ubago G, García-Portilla MP, Grupo de Trabajo sobre la Salud Física del Paciente con Esquizofrenia. Consenso sobre la salud física del paciente con esquizofrenia de las Sociedades Españolas de Psiquiatría y de Psiquiatría biológica. Actas Esp Psiquiatr. 2008;36(5):251-264.

(43) Lehman AF, Lieberman JA, Dixon LB, McGlashan TH, Miller AL, Perkins DO, et al. Practice guideline for the treatment of patients with schizophrenia, second edition. Am J Psychiatry. 2004 Feb;161(2 Suppl):1-56.

(44) Canadian Psychiatric Association Working Group. Clinical practical guidelines: Treatment of Schizophrenia. Can J Psychiatry. 2005 Nov;50(13 Suppl 1):7S-57S.

(45) American Diabetes Association, American Psychiatric Association, American Association of Clinical Endocrinologists, North American Association for the Study of Obesity. Consensus develo- 
pment conference on antipsychotic drugs and obesity and diabetes. Diabetes Care. 2004 Feb;27(2):596601.

(46) Tamminga CA, Bellack AS, Marder SR, Fenton WS. Psicofarmacología clínica y restauración cognitiva. En: Gabbard GO. Tratamiento de los trastornos psiquiátricos. Ars XXI; Barcelona: 2007. p. $315-331$ 